\title{
Computational Study of Damage Profiles and Energy Loss of Gallium Ion in Germanium Substrate
}

\section{K. Giri and A. Bhandari}

Journal of Nepal Physical Society

Volume 6, Issue 1, June 2020

ISSN: 2392-473X (Print), 2738-9537 (Online)

Editors:

Dr. Binod Adhikari

Dr. Manoj Kumar Yadav

Mr. Kiran Pudasainee

JNPS, 6 (1), 117-122 (2020)

DOI: http://doi.org/10.3126/jnphyssoc.v6i1.30560

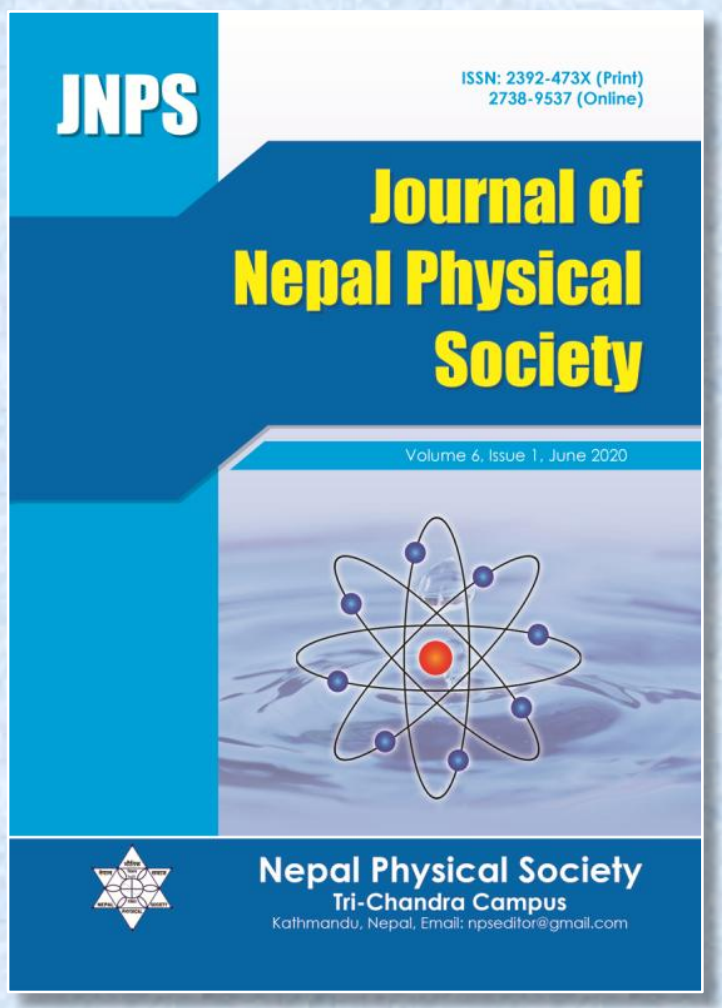

Published by:

Nepal Physical Society

P.O. Box: 2934

Tri-Chandra Campus

Kathmandu, Nepal

Email: npseditor@gmail.com 


\title{
Computational Study of Damage Profiles and Energy Loss of Gallium Ion in Germanium Substrate
}

\author{
K. Giri ${ }^{1, *}$ and A. Bhandari ${ }^{2}$ \\ ${ }^{1}$ Department of Physics, Tri-Chandra Multiple Campus, Kathmandu, Nepal \\ ${ }^{2}$ Department of Physics, Tri-Chandra Multiple Campus, Tribhuvan University, Kathmandu, Nepal \\ *Corresponding Email: karangiri575@gmail.com
}

Received: 21 Jan., 2020; Revised: 16 May, 2020; Accepted: 24 Jun., 2020

\begin{abstract}
Computational calculation of energy loss and study of damage profiles during ionic implantation by gallium ions on germanium had been carried out. The required energies for doping of gallium ion on germanium, in order to obtain maximum damage at $600 \AA$, were calculated using SRIM; Stopping and Range of Ions in Matter. The ions when implanted independently on germanium causes the production of germanium recoils, vacancy-interstitial pairs, and phonons during the collision process. For $130 \mathrm{keV}$ gallium ion, the energy used for ionization, phonon production and vacancies creation are $37.713 \mathrm{keV}$ (29.01\% of incident energy), $90.006 \mathrm{keV}$ (64.29\% of incident energy) and $8.71 \mathrm{keV}$ (6.7\% of incident energy) respectively. The amount of target displacement, replacement collisions and vacancies were also evaluated. Doping of gallium ions on germanium also reveals that the energy loss due to nuclear stopping was greater than electronic stopping.
\end{abstract}

Keywords: Doping, Implantation, Ionization, Nuclear stopping, Phonons, Target displacement.

\section{INTRODUCTION}

\subsection{Ion Implantation}

This study presents the computational calculations of energy loss and damage profiles during ion implantation on germanium $(\mathrm{Ge})$ substrate. When a beam of energetic gallium ions is directed to $\mathrm{Ge}$ substrate, it will not only lose some of its own ions by sputtering but will also retain some of the incident ions. The incident ions that are clung are said to have been implanted, and the method of using an high energy ion beam to introduce ions into a substrate is called ion implantation. The interaction of a host lattice with the energetic beams develops metastable states and structures which can be acquired by ion implantation [1]. Ion implantation in semiconductors is presently gaining attention in several rather different situations. Semiconductor device engineers and physicists are enthusiastic in the implantation technique because it provides a new doping technique with several potential benefits over more traditional doping methods. Moreover, nuclear physicists are attentive in the ion distribution profile in an implanted semiconductor substrate because, among other things, it produces essential information on the nature of the physical methods that occur when a crystalline or amorphous target is bombarded with an energetic particle [2]. At the present days the ion implantation has become easier to a great extent by different simulations and computer programming like SRIM. It is specifically suitable for investigation of the effect of beam parameters on the ion distributions in the substrate [3]. In SRIM thermal effects are not considered for calculation of damage profiles on germanium because ion implantation is a low temperature process. The damage which is calculated is that which would happen for an implantation at $0 \mathrm{~K}$.

\subsection{Germanium as a Substrate for Ion Implantation}

The germanium semiconductor, atomic number 32, has much higher holes and electrons mobility than other semiconductors in its intrinsic state. It has intermediate electrical conductivity lies between that of a conductor and an insulator [4]. We are interested in analysis of damage profiles of germanium substrate at the range of $600 \AA$ when doped with p-type gallium ions. 


\subsection{Stopping Power}

When ions impinges matter then an important parameter, stopping power, comes into play. It is defined as how much energy per unit path length an ion imparts when penetrating a matter or crystal. It is in general measured in units of $\mathrm{eVcm}^{-1}$ but here we have expressed the stopping power in eV/A. When the target substrate is penetrated by energetic ions a series of screened coulomb collision comes into play where the ion's energy gets divided into two stopping parameters, one is nuclear stopping and another is electronic stopping. Mathematically, the total stopping power (S) can be expressed as:

$\mathrm{S}=\left(\frac{d E}{d x}\right)_{\text {nuclear }}+\left(\frac{d E}{d x}\right)_{\mathrm{e}}$

Total stopping power is defined as the energy loss $(E)$ per unit path length $(x)$ of the ion and is the sum of two factors;

nuclear stopping, $\left(\frac{d E}{d x}\right)_{\text {nuclear }}$ and

electrical stopping, $\left(\frac{d E}{d x}\right)_{\text {electronic }}$

Nuclear stopping is the energy loss by ion to the target nuclei per unit path length of the ion. It is an elastic collision between two atoms and can be described by classical kinematics. Electronic stopping is the energy loss by ion to the electrons of target atoms per unit path length. It is an inelastic collision and the theoretical model is quite complex. The stopping is identical to a drag force; viscous force and is proportional to the velocity of ion. The energy imparted by incident ions is lost through the electron cloud in the form of thermal vibrations of the target substrate. The energy imparted by electronic energy loses are more essential than nuclear energy loses for upgrading hardness at the near-surface region. The hardness and electrical conductivity enhances with the increase in electronic energy loss while, conductivity and hardness enhances with the reduction in nuclear energy loss. This remarkable up gradation in hardness and electrical conductivity is correlated to the extent of radiation-induced changes in microstructure and composition. Specifically, determination of the electronic stopping power becomes crucial for studies of ion ranges and radiation damage in semiconductor substrates.

\section{METHOD AND SIMULATION DETAIL}

The doping of impurities on semiconducting substrates can be prepared by two methods; diffusion and implantation methods [5]. Diffusion is limiting technique due to saturation limit and therefore, we are unable to make high concentrated carriers. To defeat the diffusion issues of dopants and activation of dopants in semiconductor substrate, ion irradiation or ion implantation is the outstanding technique. In general, to bombard energetic ion on a semiconductor material particle accelerator is employed and it is the basic process of implantation and irradiation. For the study of damage profiles of target atom doped with different ions SRIM is one of the trending software. Furthermore, energy loss of proton beam on ovary tumor by K. Giri and R. Khanal galvanize to work on this research work. We employ same methods in different prospects [6].

\subsection{Simulation Methodology}

The applied simulation software for the ion implantation is SRIM and MATLAB. SRIM features around 28,000 built-in experimental data for stopping power and therefore allows for accurate simulation of ion penetration into matter [7]. The essence of SRIM is a Program Transport of Ions in Matter (TRIM). When an ion impinges the target material, it undergoes sequences of screened coulomb collisions uprooting target atoms along the way. TRIM will follow atoms endlessly as they go sideways, although they leave the screen. But if they pass through either target surface they are discarded and not counted. In fact, SRIM calculates the interactions of energetic ions with amorphous targets and presents those interactions statistically and graphically which make the implantation process easier to understand.

Using a quantum mechanical treatment of ion-atom SRIM calculates the stopping and range of ions (10 $\mathrm{eV}$ - $2 \mathrm{GeV} / \mathrm{amu})$ into target material. During bombardments, the atom and ion have a screened Coulomb collision, including exchange and correlation interactions between the overlapping electron shells. The target atoms are allowed to have long range interactions with the ion creating electron excitations and plasmons within the target. It can also constitutes three-dimensional plots of the ion distribution when penetrate into matter, including the trace of defects produced by the ion beam and its so-called straggle and all resulting cascades which can occur when atoms are kicked out of their lattice position, becoming an interstitial atom which can subsequently hit other lattice atoms [8]. MATLAB is now also used in education, specifically the instruction of linear algebra, 
numerical analysis, and is trending amongst analyst involved in image processing [9].

\subsection{Range Distribution}

The range distribution depends primarily on the energy, mass, and atomic number of the incoming ions, the mass and atomic number of target atoms, the density of the target. Both the number of collisions and the energy transferred per collision are random variables, all ions of a given type and initial energy will not have the same range. In some degree, there should be a distribution of stopping points in space, or a range distribution, which are characterized by quoting a mean ion range and perhaps higher moments of the distribution function like skewness, kurtosis.

\section{RESULTS AND DISCUSSION}

\subsection{Energy Calculations}

The energy needed to implant gallium ions on germanium in order to obtain peak dopants concentration at range of $600 \AA$ were tabulated using SRIM. We have the target density $=5.350$ $\mathrm{g} / \mathrm{cm}^{3}=4.4371 \times 10^{22}$ atoms $/ \mathrm{cm}^{3}$. Table 1 demonstrates that $130 \mathrm{keV}$ energy is required for gallium to have the projected range of $592 \AA$ in germanium which is close enough to our estimated range, so we can now implant 10,000 gallium

Table 1: Energy Loss for Gallium Ion

\begin{tabular}{|c|c|c|c|}
\hline $\begin{array}{c}\text { Ion } \\
\text { Energy } \\
\text { (keV) }\end{array}$ & $\begin{array}{c}\text { dE/dx } \\
\text { Electrical } \\
\text { Stopping }\end{array}$ & $\begin{array}{c}\mathbf{d E / d x} \\
\text { Nuclear } \\
\text { Stopping }\end{array}$ & $\begin{array}{c}\text { Projected } \\
\text { Range (̊) }\end{array}$ \\
\hline 70.00 & 17.81 & 144.2 & 342 \\
\hline 80.00 & 19.04 & 144.6 & 383 \\
\hline 90.00 & 20.19 & 144.7 & 425 \\
\hline 100.00 & 21.29 & 144.4 & 466 \\
\hline 110.00 & 22.33 & 143.9 & 508 \\
\hline 120.00 & 23.32 & 143.3 & 550 \\
\hline $\mathbf{1 3 0 . 0 0}$ & $\mathbf{2 4 . 2 7}$ & $\mathbf{1 4 2 . 6}$ & $\mathbf{5 9 2}$ \\
\hline 140.00 & 25.43 & 141.7 & 634 \\
\hline 150.00 & 27.31 & 140.8 & 677 \\
\hline 160.00 & 28.99 & 139.9 & 719 \\
\hline 170.00 & 30.52 & 138.9 & 761 \\
\hline 180.00 & 31.95 & 137.9 & 804 \\
\hline 200.00 & 34.53 & 135.8 & 889 \\
\hline
\end{tabular}

ions accelerated by $130 \mathrm{keV}$ energy in germanium monolayer target in order to obtain maximum defects concentration at $600 \AA$.

\subsection{Stopping Power}

Fig.1 demonstrates the nature of stopping power for $\mathrm{Ga}$ ions bombarded on germanium. On increasing ion's energy nuclear stopping goes on increasing upto $90 \mathrm{keV}$ and then gradually drops out while electronic stopping goes on gradual increase and starts dominating nuclear energy loss above 90 $\mathrm{keV}$. The point of intersection of the two curves represents the corresponding ion energy where the electronic and nuclear energy losses are equal. As mentioned by K. Giri and R. Khanal [6] for the human ovary the nuclear interaction has insignificant contribution on total stopping power of the medium. The major loss of energy is caused by electronic interaction of proton i.e. ionization. For $130 \mathrm{keV}$ gallium ions, $24.27 \mathrm{eV} / \AA$ and 142.60 $\mathrm{eV} / \AA$ are the value of electronic stopping and nuclear stopping respectively. These values indicate that in average each gallium ion lose $24.27 \mathrm{eV} / \AA$ to target electrons for every unit path of ion, whereas, to the target atoms, each gallium ion lose 142.60 $\mathrm{eV} / \AA$ for every unit path of ion. Since for gallium ion accelerated at $130 \mathrm{keV}$ have greater nuclear stopping so there is no improvement in surface hardness and electrical conductivity. The reason behind dominance of nuclear stopping is mass of ions that are comparable with germanium. Ion's energy should be increased to energy above $1 \mathrm{MeV}$ for $\mathrm{Ga}$ ions in order to obtain dominance of electronic stopping power over nuclear stopping

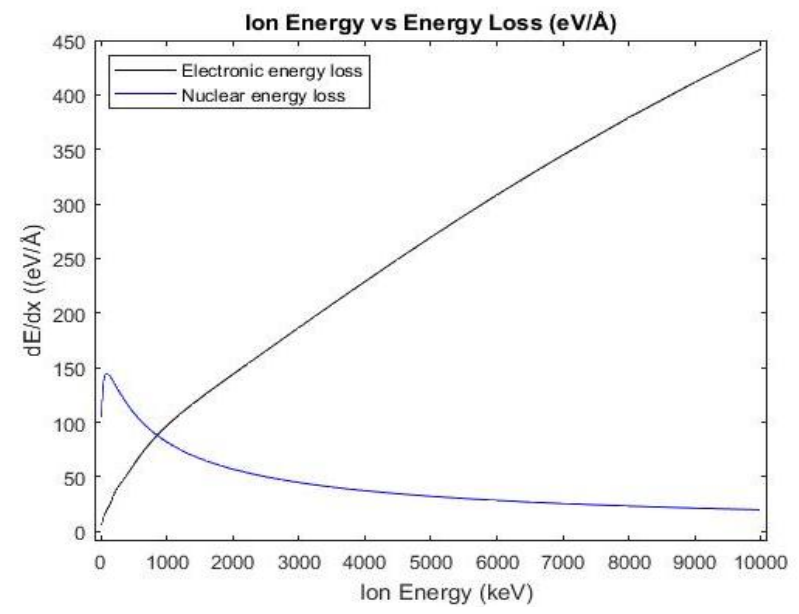

Fig.1: Comparison of electronic energy loss and nuclear energy loss for gallium ions 
power that eventually improve the surface hardening and electrical conductivity. However, study of damage profiles for tens of mega electron volt is not included in this work.

\subsection{Energy Loss during Ionization}

Fig.2 reveals the energy imparted to the target electrons during ionization process. The plot shows ionization from the incident ions and from recoiling target atoms. The energy loss to target electrons by recoil atoms is greater than energy loss by ions. As suggested by K. Giri and B. Kandel [10] maximum ionization by ions begins from the surface of the target material and then gradually decreases. Energy loss by gallium ions and recoil atoms during ionization process is $11.25 \%(14.625 \mathrm{keV})$ and $17.76 \%(23.088 \mathrm{keV})$ of incident energy respectively. Total energy loss during ionization process during gallium-germanium interaction is $37.713 \mathrm{keV}$.

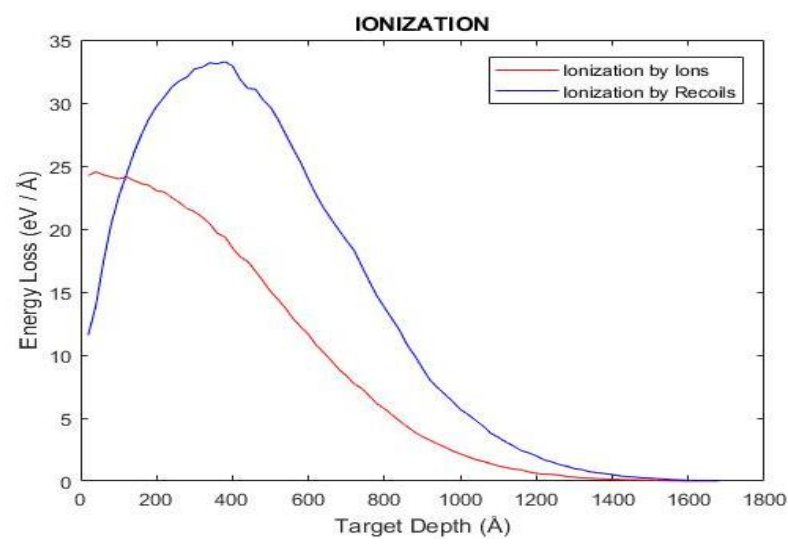

Fig. 2: Energy loss distribution during ionization

\subsection{Energy Imparted to Phonons}

Phonons are energy stored in atomic vibrations in a crystal. When a germanium atom is knocked out of its lattice site, its binding energy, $\mathrm{E}_{\text {latt }}=2 \mathrm{eV}$, is deposited into phonons produced by the recoils. Vacancies produced by each gallium ion are 4344.6. So for each gallium ion, displacements by the ion or recoil cascades cause $4344.6 \times 2 \mathrm{eV}=$ $8689.2 \mathrm{eV}$ of phonons. In Fig.3, we can barely see the energy imparted to phonons by the ions (red line at the bottom of the plot), and the phonons are produced almost exclusively by the recoiling target atoms. The energy loss for phonon production is high around the range of $400 \AA$ and then the gradually decreases as the target depth increases. The gallium ions generate phonons with $0.434 \mathrm{keV}$;
$0.31 \%$ of their incident energy, and the recoiling atoms contribute an additional $89.572 \mathrm{keV} ; 63.98 \%$ of incident energy. And total energy loss is 90.006 $\mathrm{keV}$. In fact phonons do not have any contribution in improving electrical conductivity but do have contribution in increasing temperature of target material during implantation process.

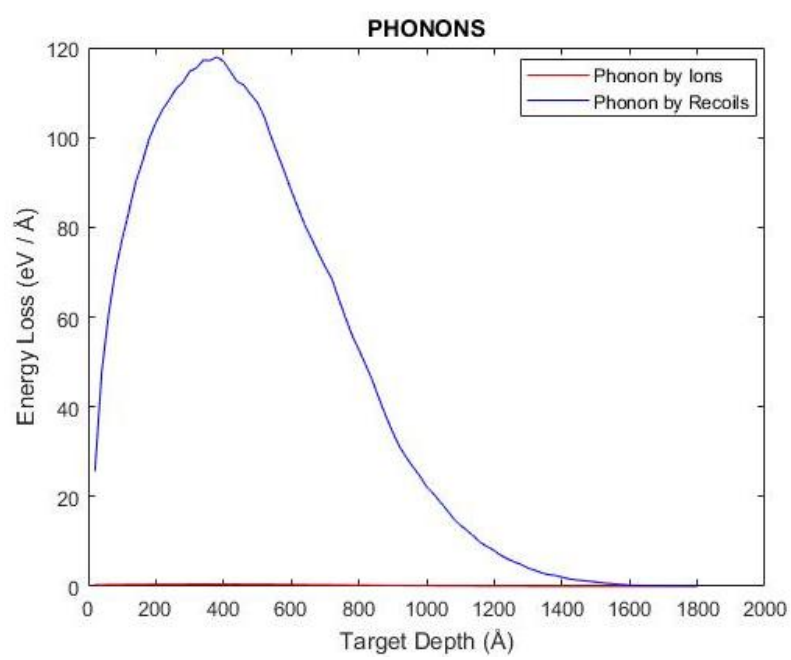

Fig.3: Distribution of energy loss to phonons

\subsection{Ion Range}

Fig. 4 is the curve of range distributions of ions building up in germanium target. The higher moments of the distribution (straggle, skewness, and kurtosis) are also mentioned in the plot. The plot has ordinate unit: (Atoms $\left./ \mathrm{cm}^{3}\right) /\left(\right.$ Atoms $/ \mathrm{cm}^{2}$ ) which might appear strange. When we multiply by an implantation dose (ions $/ \mathrm{cm}^{2}$ ), we will end up

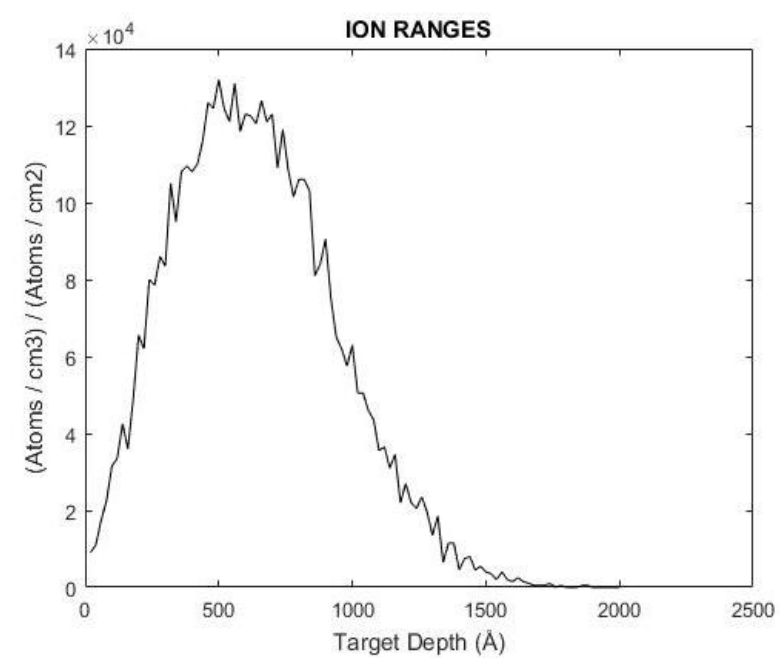

Fig. 4: Ion Range Distribution 
Table 2: Mean range and moments for Gallium

\begin{tabular}{|l|c|}
\hline Ion Range & $617 \AA$ \\
\hline Straggle & $294 \AA$ \\
\hline Skewness & 0.3300 \\
\hline Kurtosis & 2.6369 \\
\hline
\end{tabular}

with the mpurity concentration (atoms $/ \mathrm{cm}^{3}$ ) vs. depth. Target depth is taken up to $1500 \AA$ in order to catch maximum number of ion in the plot. Using Table 2, the mean ranges of implanted ions were found to be $617 \AA$ for Gallium ion. This value shows little discrepancy with the value given by SRIM. Here, $592 \AA$ was the mean ion range value tabulated by SRIM, however as per Transport of Ions in Matter (TRIM); the value was $617 \AA$. Therefore, the percentage error in ion range value was found to be $4.22 \%$ and is responsible for skewness. Moreover, this value indicates that the peak concentrations of defects are formed at range of $617 \AA$ for $130 \mathrm{keV}$ accelerated gallium. The skewness reveals whether the peak is skewed towards the surface (negative values) or away from the surface (positive values). It means the negative value of skewness indicates that the most probable depth (the peak position) is greater than the mean depth, and the positive value indicate that the reverse. The value of skewness for gallium ions is 0.330 . It says that the mean depth is greater than the most probable depth. Kurtosis indicates the extent of the distribution tails, values from 0 to 3 indicate abbreviated tails, and values above 3 indicate broad tails. The value of kurtosis is 2.6369 for gallium which is smaller than 3 and hence has the abbreviated tails. In addition, the curve is platykurtic.

\subsection{Ge recoil distribution}

Fig. 5 demonstrates that distribution of recoil atoms over target layer. The curve shows that the concentration of recoil atoms at the surface is about $1.75 \times 10^{8}$ atoms $/ \mathrm{cm}^{2}$ with the rapid increase in concentration upto $400 \AA$ followed by the gradual decrease in concentration of recoil atoms until the tail of curve. Maximum concentration of $6.75 \times 10^{8}$ atoms $/ \mathrm{cm}^{2}$, of recoil atoms can be seen at $400 \AA$.

\subsection{Lateral Distribution}

In the Table 3, we can see that the value of lateral projected range for gallium is $203 \AA$ which is the average value of 10000 implanted ions.

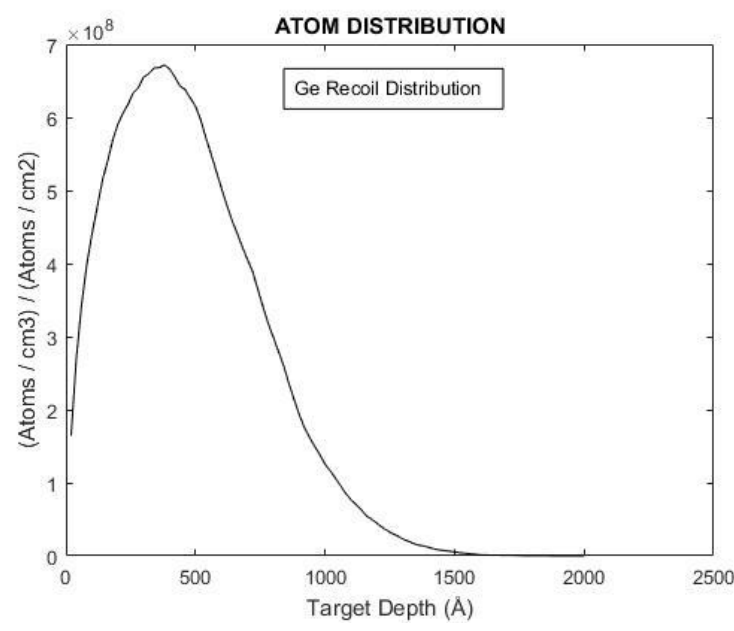

Fig. 5: Germanium recoil distribution curve

Table 3: Longitudinal, Radial and Lateral projection range for $\mathrm{Ga}$ ions

\begin{tabular}{|l|l|}
\hline Longitudinal & 617 \\
\hline Lateral Projection & 203 \\
\hline Radial & 321 \\
\hline
\end{tabular}

\subsection{Collision Events}

\subsubsection{Target Displacement}

Fig. 6 shows the total target displacement which is the number of atoms knocked off their target lattice site. Area under the curve calculations gives the values for target displacements of 4689/ion for Ga.

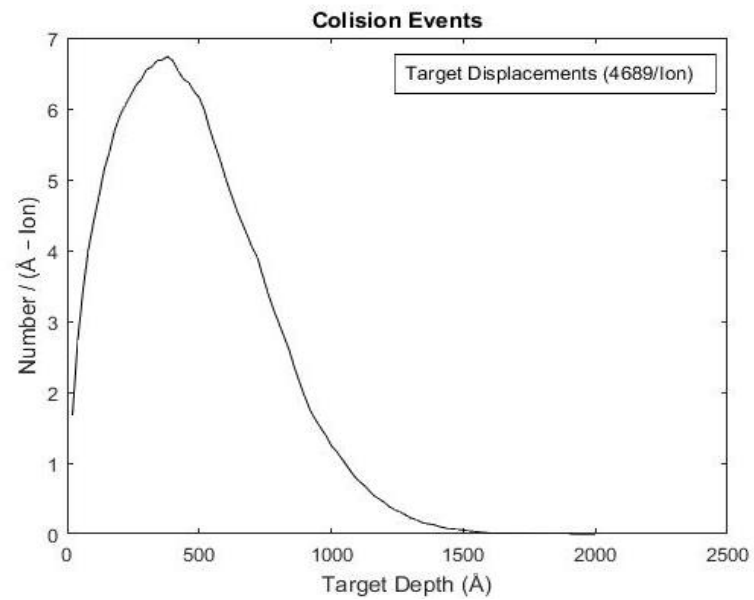

Fig.6: Curve showing target displacement by gallium ion with $130 \mathrm{keV}$

\subsubsection{Replacement Collision}

The replacement collisions of 347/ion are calculated from the collision of $\mathrm{Ga}$ ions with $\mathrm{Ge}$ 
atoms. The replacement collisions amount to $7.99 \%$ for Ga. A summary of the collision events is shown in Table 4.

Table 4: Defects produced during ion implantation of $\mathbf{G a}$ in $\mathbf{G e}$

\begin{tabular}{|l|c|}
\hline & Gallium \\
\hline Target displacements/ion & 4689 \\
\hline Vacancies/ion & 4344 \\
\hline Interstitials/ion & 4344 \\
\hline Replacement collisions/ion & 347 \\
\hline
\end{tabular}

While studying collision events, it is important to note that,

Displacements $=$ Vacancies + Replacement Collisions

For gallium-germanium collision, sum of vacancies/Ion and replacement/ion should have been equal but simulation data deviates by $2 /$ ion i.e. sum of vacancies/ion (4344/ion) and replacement collision/ion (347/ion) is not equal to target displacement (4689/ion). It means that the cascade atom leaves the target volume by $2 /$ ion which are no longer followed by TRIM. The results presented in the figures belonging to energy loss by ionization, recoil collisions, displacement collisions, and vacancies are similar by nature of graph but are characterized by difference in their numerical values depending on the type of implanted ion.

\section{CONCLUSIONS}

This fast and precise preliminary investigation demonstrates that SRIM program have great potential in quantitative analysis of doping of impurities in semiconductor. Doping of gallium ions on germanium revealed that most of the energy loss is due to ionization and phonons production. For $130 \mathrm{keV}$ gallium ion, the energy used for ionization, phonon production and vacancies creation are $37.713 \mathrm{keV}(29.01 \%), 90.006 \mathrm{keV}$ $(64.29 \%)$ and $8.71 \mathrm{keV}$ (6.7\%) respectively. Almost three fold amount of energy was lost during phonon production for $\mathrm{Ga}$ ions when implanted on germanium.

The collision events revealed the formation of interstitial vacancy pairs 4344 for Ga ions, target displacements of 4689/ion for $\mathrm{Ga}$, and replacement collisions of 347/ion are calculated from the collision of $\mathrm{Ga}$ ions with $\mathrm{Ge}$ atoms, respectively. The replacement collisions amount to $7.99 \%$ for $\mathrm{Ga}$. Another important finding of this work is we cannot improve electrical conductivity and hardness of germanium by implanting $130 \mathrm{keV}$ gallium ions. To improve these properties by implanting gallium ions we need to accelerate $\mathrm{Ga}$ ions above $1 \mathrm{MeV}$.

\section{ACKNOWLEDGEMENT}

Authors acknowledge editor and referee for their constructive and innovative comments and suggestions. We express our thanks to Nepal Physical Society and Department of Physics, TriChandra Multiple Campus for their support.

\section{REFERENCES}

[1] Picraux, S.T. and Peercy P.S., Ion Implantation of Surfaces. Scientific American, 252, 102-104 (1985).

[2] Gibbons, J.F., Ion Implantation in Semiconductors-Part I: Range Distribution Theory and Experiments. Proceedings of the IEEE. 56 (3), 295-319 (1968).

[3] Pavlovic, M. and Strašík, I.: Supporting Routines to the SRIM Codes, Nucl. Instr. and Meth. B 257, 601-604 (2007)

[4] Neamen, D., Semiconductor Physics and Devices, McGraw-Hill, West 19th Street New York, NY United States (2002).

[5] Bruel, M., Floccari, M., inventors; Commissariat and 'Energie Atomique, Assignee. Process for doping semiconductors. United States patent 4, 368-383 (1983).

[6] Giri, K. and Khanal R., Journal of Nepal Physical Society 5(1), 24-29 (2019).

[7] James F. Ziegler, M.D. Ziegler, J.P. Biersack,The stopping and range of ions in matter, Nuclear Instruments and Methods in Physics Research Section B: Beam Interactions with Materials and Atoms, 268 (11-12),1818-1823 (2010)

[8] Ziegler, J.F., Biersack, J. and Littmark, U., “'The Stopping and Range of Ions in Matter', Pergamon Press, New York (1985).

[9] Moler C. the Creator of MATLAB (2004).". The Origins of MATLAB". Retrieved on April. (2007).

[10] Giri K. and Kandel B., Journal of Physical Sciences, BIBECHANA, 17, 96-103 (2020). 